\title{
Capacidade de redes ad hoc e mesh sem fio
}

\author{
Fabrício Lira Figueiredo, Paulo Cardieri e Álvaro Augusto Machado de Medeiros
}

\begin{abstract}
Resumo- Este artigo apresenta um método semianalítico para o dimensionamento de capacidade de redes ad hoc e mesh sem fio. A partir de um modelo analítico que representa o comportamento das camadas física, de enlace de dados e de roteamento, é construída uma ferramenta de simulação estatística que provê métricas relacionadas à capacidade destas redes. São apresentados exemplos de aplicação do método, calculando a capacidade por nó em função de parâmetros como densidade de gateways e raio de cobertura, bem como o posicionamento ótimo dos gateways. Ao final, uma metodologia de dimensionamento utilizando o método é proposta.
\end{abstract}

Palavras-Chave- Redes Ad hoc, Redes Mesh Sem Fio, Capacidade.

Abstract - This article presents a semi-analytical method for capacity dimensioning of wireless ad hoc and mesh networks. From an analytical method that represents the physical, MAC and routing layers, a statistical simulator is designed, which provides metrics related to capacity. Examples of application of the proposed method are presented. Finally, a dimensioning methodology based on the proposed method is proposed.

Keywords - Ad hoc Networks, Wireless Mesh Networks, Capacity.

\section{INTRODUÇÃO}

O dimensionamento da capacidade de redes ad hoc ou mesh sem fio tem sido importante objeto de pesquisa nos últimos anos. Além dos aspectos teóricos, a construção de metodologias consistentes para a estimativa de capacidade tem grande relevância prática, pois tem aplicação direta na implantação e otimização destas redes.

Diversas abordagens têm sido adotadas para investigar este tema específico em redes ad hoc e mesh sem fio, cujo objetivo principal é determinar limites teóricos superiores e inferiores de capacidade, em diferentes condições. Estes resultados refletem principalmente uma relação entre capacidade e algum parâmetro de interesse, como, por exemplo, o número de nós. Contudo, se baseiam em um conjunto de

Fabrício L. Figueiredo e Álvaro A. M. de Medeiros, Gerência de Comunicações Sem Fio, Fundação CPqD, Rod. SP-340, km 118,5, Campinas - SP, 13086-902, email: [fabricio,amachado]@cpqd.com.br. Paulo Cardieri, Wireless Technology Laboratory (Wisstek) DECOM/FEEC/UNICAMP - C.P. 6101, Campinas - SP, 13081-970, e-mail: cardieri@wisstek.org. Este trabalho foi financiado pela Fundação $\mathrm{CPqD}$ e FUNTTEL. O trabalho de Paulo Cardieri foi parcialmente financiado pelo CNPq Proc. No. 312535/2006-6. premissas que muitas vezes não se aplicam em cenários reais. Em [1], mostra-se que, para nós estáticos e agendamento ideal de transmissões, a taxa de transmissão por nó é limitada por uma função inversamente proporcional à raiz quadrada do número de nós. Resultados similares baseado em simulação são apresentados em [2]. Também através de simulações, os limites de capacidade das redes mesh são estudados em [3]. Tal estudo indica que o limite da capacidade total da rede decai com o inverso da quantidade de nós, o que é um resultado pior do que aquele apresentado em [1], visto que o tráfego das redes mesh originam ou terminam em um gateway. Em [4], são analisadas as interações entre as camadas física (PHY) e de acesso ao meio (MAC) para estimar a vazão de todas as transmissões simultâneas para, então, somá-las, resultando na capacidade total.

Neste artigo é apresentado um modelo para o dimensionamento de capacidade de redes ad hoc e mesh sem fio, baseado em uma abordagem semianalítica. A partir de um modelo analítico que representa o comportamento das camadas física, de enlace de dados e de roteamento em topologias ad hoc e mesh, é construída uma ferramenta de simulação estatística que provê métricas relacionadas à capacidade destas redes. Esta abordagem elimina a necessidade de adoção de ferramentas de simulação a eventos discretos, permitindo a obtenção de resultados mais gerais com menor esforço computacional. O modelo é validado através de comparações com uma ferramenta de simulação a eventos discretos. São apresentados também resultados de análise do comportamento da rede quando são inseridos os gateways para o escoamento do tráfego e considerando as localizações destes na malha de nós que proporcionam a máxima capacidade para os usuários.

\section{Modelo de Estimação da CAPacidade}

O modelo proposto neste artigo para o dimensionamento de capacidade de redes ad hoc e mesh sem fio busca representar a estrutura de múltiplas camadas referente à arquitetura de protocolos destas redes, que compreende a camadas física, de enlace de dados, de roteamento e de tráfego. O modelo baseia-se em um algoritmo 
que permite dimensionar a máxima capacidade por usuário $G_{\max }$ que pode ser proporcionada em uma dada topologia $\mathfrak{I}$, para uma determinada densidade de nós e gateways. $\mathrm{O}$ modelo proposto compreende as seguintes entradas:

- Número e localização de nós e gateways na área a ser coberta pela rede;

- Parâmetros da interface de rádio, em conformidade com o padrão IEEE 802.11 [5];

- Comprimento em bits dos pacotes de dados e de sinalização;

- Caracterização de protocolos de roteamento ad hoc (opcional).

A seguintes saídas são fornecidas pelo modelo:

- Identificação de nós ou enlaces sobrecarregados (gargalos);

- A máxima banda disponível por usuário para garantir disponibilidade do serviço, considerando cenários de pior caso;

- Variação da capacidade com o número de nós e o número de gateways;

- Posicionamento dos gateways que maximiza a banda disponível por usuário;

- Dimensionamento de quantidade e capacidade dos gateways;

- Comprimento médio das rotas estabelecidas.

Os principais passos do algoritmo são descritos a seguir. Antes, porém, algumas definições se fazem necessárias:

- $\rho_{n}$ : densidade de nós distribuídos uniformemente em uma área, em nós $/ \mathrm{km}^{2}$;

- $\quad N$ : número de nós distribuídos uniformemente em uma área $A$, dado por $\rho_{n} A$;

- $\quad \vec{p}_{n}$ : matriz composta pelas coordenadas $(x, y)$ dos nós $\left\{n_{i}\right\}_{i=1}^{N}$ da topologia, de dimensão $N \mathrm{x}$ 2 ;

- $\quad \rho_{g w}$ : densidade de gateways distribuídos em uma área, em gateways $/ \mathrm{km}^{2}$;

- $\quad N_{g w}$ : número de gateways distribuídos uniformemente em uma área $A$, dado por $\rho_{g w} \cdot A$;

- $\quad \vec{p}_{g w}$ : matriz composta pelas coordenadas $(x, y)$ dos gateways $\left\{g w_{i}\right\}_{i=1}^{N_{g w}}$ da topologia, tendo dimensão $N_{g w}$ x 2 .

\section{A. Cálculo da Matriz de Conectividade}

$\mathrm{O}$ primeiro passo do algoritmo consiste em determinar a matriz de conectividade $C=\left\{c_{i j}\right\}_{i, j=1}^{N}$, que representa a existência de enlaces entres os nós da topologia. Os elementos $c_{i j}$ são definidos como:

$$
c_{i j}=\left\{\begin{array}{ll}
1, \text { se } & P r_{i j} \geq P r_{\text {sen } s} \\
0, \text { se } & P r_{i j}<P r_{\text {sen } s}
\end{array},\right.
$$

em que $P r_{i j}$ é a potência no receptor do nó $n_{j}$ considerando transmissão a partir do nó $n_{i}$, e $P r_{\text {sens }}$ corresponde à sensibilidade de recepção, definida em conformidade com o padrão IEEE 802.11. A potência $P r_{i j}$ recebida no nó destino $n_{j}$ do sinal transmitido pelo nó $n_{j}$ é calculada com base na perda no sistema $L s_{i j}$, a qual é estimada com base em um modelo que caracterize o ambiente de propagação, resultando em

$$
P r_{i j}=P t_{i}+G s_{i j}-L s_{i j}[d B m],
$$

em que $G s_{i j}$ é o ganho total do sistema.

\section{B. Construção de conjunto de gateways candidatos}

Em seguida, visando minimizar a complexidade do algoritmo, é pré-estabelecido um conjunto de gateways candidatos $\vec{p}_{g w c}$, sobre o qual será realizada a busca pelo sub-conjunto ótimo de gateways $\vec{p}_{g w}$, que maximiza a capacidade da rede. Para tanto, a área $A$ da topologia é particionada em $N_{s a}$ sub-áreas disjuntas e iguais, resultando no conjunto $\left\{A_{i}\right\}_{i=1}^{N s a}$. Para cada sub-área $A_{i}$, um único nó é selecionado dentre os nós que pertencem a esta região como gateway candidato $g w_{i}$. Vários critérios podem ser utilizados para selecionar o nó candidato, mas foi verificado, através de simulações, que o critério de máxima conectividade proporciona uma boa estratégia para se obter a capacidade máxima por usuário $G_{\max }$ do sistema. O critério de máxima conectividade é descrito por $g w_{i}=\arg \max _{\forall n \in A_{i}}\left|\vec{V}_{1}(n)\right|$ em que $\vec{V}_{1}(n)$ é um vetor contendo os identificadores dos nós vizinhos imediatos do nó $n$ e $\left|\vec{V}_{1}(n)\right|$ é o número de elementos de $\vec{V}_{1}(n)$. O conjunto de gateways candidatos é definido como $\vec{p}_{g w c}=\left\{\vec{p}_{g w_{i}}\right\}_{i=1}^{N_{g w c}}$, em que $N_{g w c}$ corresponde ao número de conjuntos candidatos não nulos, sendo, portanto, menor ou igual a $N_{s a}$.

\section{Construção Matriz de Rotas}

Este passo do algoritmo consiste em construir a matriz de rotas $R\left(\vec{p}_{n}, \vec{p}_{g w}\right)$ da topologia, cujas linhas correspondem a um vetor que representa o caminho entre os nós $\vec{p}_{n}$ e os gateways candidatos $\vec{p}_{g w c}$, a qual é definida por 


$$
R\left(\vec{p}_{n}, \vec{p}_{g w}\right)=\left\{\vec{r}_{k}\left(\left[\vec{p}_{n}\right],\left[\vec{p}_{g w}\right]\right)\right\}_{k=1}^{N_{r}},
$$

em que $\left[\vec{p}_{n}\right]$ e $\left\lfloor\vec{p}_{g w}\right\rfloor$ representam os elementos dos vetores $\vec{p}_{n}$ e $\vec{p}_{g w c}$, respectivamente, $\vec{r}_{k}\left(n_{s}, n_{d}\right)$ é uma rota entre os nós $n_{d}$ e $n_{s}, N_{r}$ é o número total de rotas entre os nós de $\vec{p}_{n}$ e $\vec{p}_{g w c}$. A rota $\vec{r}_{k}\left(n_{s}, n_{d}\right)$ pode ser expressa como uma seqüência de nós ou enlaces, de acordo com o formato

$$
\left.\vec{r}_{k}\left(n_{s}, n_{d}\right)=\left[n_{s}, n_{1}, \ldots, n_{L-1}, n_{d}\right]=\mid l_{s n_{1}}, l_{n_{1} n_{2}}, \ldots, l_{n_{L-1} n_{d}}\right\rfloor,
$$

em que $n_{i}$ corresponde ao $i$-ésimo nó intermediário da rota e $l_{n_{i} n_{j}}$ corresponde ao enlace entre os nós $n_{i}$ e $n_{j}$.

Uma rota $\vec{r}\left(n_{s}, n_{d}, C\right)$ é definida como o caminho ótimo entre os nós $n_{s}$ e $n_{d}$, segundo uma métrica de roteamento específica e sobre uma topologia com matriz de conectividade $C$. Um critério típico é o caminho mais curto (shortest-path), que é tipicamente empregado por protocolos de roteamento do tipo linkstate [7]. Outro critério corresponde ao caminho de menor atraso, normalmente usado por protocolos reativos [7], isto é

$$
\vec{r}\left(n_{s}, n_{d}, C\right)=\arg \min _{\forall \vec{r}_{i}\left(n_{s}, n_{d}, C\right)} \delta\left(\vec{r}_{i}\left(n_{s}, n_{d}, C\right)\right),
$$

em que $\delta(\vec{r})$ corresponde ao atraso fim-a-fim sobre uma rota $\vec{r}$, calculado como a soma dos atrasos dos enlaces que a compõem, usando

$$
\delta(\vec{r})=\delta_{s}+\delta_{d}+\sum_{k=1}^{L-1} \delta\left(n_{k}\right)+\sum_{k=1}^{L-2} \delta\left(l_{n_{k} n_{k+1}}\right),
$$

em que $\delta_{s}$ é o atraso no nó origem, $\delta_{d}$ é o atraso no nó destino, $\delta\left(n_{k}\right)$ é o atraso no nó $n_{k}$ e $\delta\left(l_{n_{k} n_{k+1}}\right)$ é o atraso de propagação no enlace $l_{n_{k} n_{k+1}}$.

Visando simplificar a busca das rotas, adotou-se um critério do menor atraso simplificado, considerando apenas o atraso de propagação nos enlaces. Neste caso, a busca pela rota ótima se baseia no algoritmo de Dijkstra, aplicado sobre a matriz de atraso de propagação $D$, definida por $D=\left\{\delta_{i j}\right\}_{i, j=1}^{N}$,

em que

$$
\delta_{i j}=\left\{\begin{array}{cc}
d_{i j} / c, s e & c_{i j}=1 \\
0, \text { se } & c_{i j}=0
\end{array},\right.
$$

no qual $c$ corresponde à velocidade de propagação da onda de rádio e $d_{i j}$ é a distancia entre os nós $n_{i}$ e $n_{j}$.

\section{Aplicação do método de Monte Carlo}

Uma vez definida a matriz de $\operatorname{rotas} R\left(\vec{p}_{n}, \vec{p}_{g w}\right)$, o próximo passo do algoritmo consiste em realizar uma busca pelo conjunto ótimo de gateways $\vec{p}_{g w}^{*}$ que maximiza a capacidade suportada por usuário para uma topologia de matriz de conectividade $C$, sendo a capacidade máxima $G_{\max }$ expressa por

$$
G_{\max }=\max _{\forall \vec{p}_{g w}=\bar{p}_{g w c}} G\left(\vec{p}_{g w}, \vec{p}_{n}, C\right),
$$

em que $G\left(\vec{p}_{g w}, \vec{p}_{n}, C\right)$ é a taxa de transmissão por nó. Neste caso, o conjunto $\vec{p}_{g w}^{*}$ é determinado por

$$
\vec{p}_{g w}^{*}=\underset{\forall \vec{p}_{g w} \sim \vec{p}_{g w c}}{\arg \max } G\left(\vec{p}_{g w}, \vec{p}_{n}, C\right) .
$$

A determinação da capacidade $G\left(\vec{p}_{g w}, \vec{p}_{n}, C\right)$ se baseia no método proposto em [3] e está descrita em [7]. Em linhas gerais, a determinação de $G\left(\vec{p}_{g w}, \vec{p}_{n}, C\right)$ consiste em encontrar o enlace gargalo da topologia e a taxa máxima que este suporta. Para estimar a capacidade $G_{\max }$, realizase, portanto, uma busca com restrições sobre todas as possíveis combinações de posicionamento de gateways em uma dada topologia, considerando que os gateways deverão ser posicionados apenas em localizações onde está prevista a presença de um nó de usuário. O pseudo-código pode ser encontrado em [7], e não é reproduzido aqui por restrições de espaço.

Finalmente, adota-se o método de Monte Carlo para calcular a capacidade média $G_{m}$, sobre um conjunto de topologias $\left\{T_{i}\right\}_{i=1}^{M}$ com nós e gateways aleatoriamente distribuídos, em função da densidade de gateways e nós nesta área, isto é

$$
G_{m}\left(\rho_{n}, \rho_{g}\right)=\frac{1}{M} \sum_{i=1}^{M} G_{\max }\left(\vec{p}_{n}, \vec{p}_{g}, C_{i}\right),
$$

em que, $\rho_{g}$ é a densidade de gateways, $M$ é a quantidade total de topologias, $C_{i}$ é a matriz de conectividade da $i$-ésima topologia.

\section{VALIDAÇÃo Do Modelo}

A validação do modelo de dimensionamento de capacidade proposto foi realizada através da comparação com resultados obtidos através da ferramenta de simulação de redes $N S-2$. Para tanto, adotaram-se os cenários de topologia aleatória descritos na Tabela I, que compreendem topologias de 25,50 e 100 nós, com 1,3 e 5 gateways posicionados de forma a maximizar a capacidade da rede. O número de topologias foi considerado de modo a garantir um intervalo de confiança de $90 \%$.

Os resultados obtidos estão mostrados na Figura 1 , para a capacidade por nó $G_{m}$. Observa-se a boa concordância dos resultados gerados através do modelo, com valores obtidos com a ferramenta NS-2. Pode-se ainda verificar a diminuição da capacidade com o aumento do número de nós, que é mais acentuada quanto maior o número de gateways, indicando que em topologias menos densas, obtém-se maior ganho de capacidade com o aumento da quantidade de gateways. 
TABELA I

Cenários de validação do modelo de CAPACidade.

\begin{tabular}{|l|l|}
\hline Número de nós & 25,50 e 100 \\
\hline Número de gateways & 1,3 e 5 \\
\hline Área de simulação & $2,5 \mathrm{~km}$ x 2,5 km \\
\hline Número de topologias & 15 \\
\hline Tempo de simulação & $300 \mathrm{~s}$ \\
\hline Modelo de propagação & Two-Ray Ground \\
\hline Raio de cobertura & $750 \mathrm{~m}$ \\
\hline Potência de transmissão & $20 \mathrm{dBm}$ \\
\hline Protocolo de roteamento & DSR \\
\hline Protocolo MAC & IEEE 802.11b \\
\hline Modelo de Tráfego & Constant Bit Rate (CBR) \\
\hline Taxa de transmissão (PHY) & 11 Mbps \\
\hline
\end{tabular}

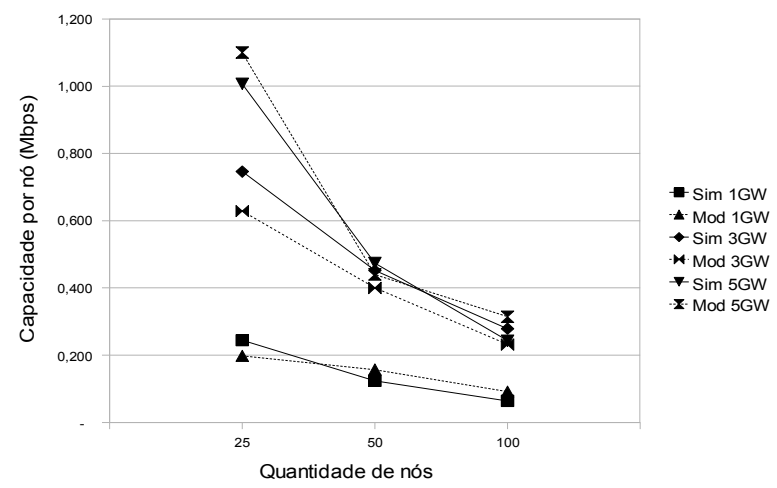

Fig. 1 - Comparação entre resultados obtidos através do modelo proposto e da ferramenta NS-2 para a capacidade por nó $G_{m}$.

\section{AnÁlise da CAPACIDADE}

Para análise da capacidade, utilizou-se o cenário descrito na Tabela I. Contudo, foi adotado como parâmetro, a densidade de nós da rede, visando eliminar a dependência em relação à área de topologia. Para tanto, no método de Monte Carlo descrito na Seção II, foram utilizadas 10 topologias aleatórias com diferentes áreas para cada densidade de nós. Esta abordagem foi adotada para evitar a dependência de um valor específico de área ocupada pela topologia, permitindo a adoção da densidade de gateways como parâmetro único de análise. Os valores para as densidades de nós utilizados foram 7,5; 12,5; 25; 37,5; 50 nós $/ \mathrm{km}^{2}$.

\section{A. Impactos do aumento da densidade de gateways}

A quantidade de gateways em uma rede mesh sem fio tem impacto significativo na capacidade média disponibilizada a cada usuário $\left(G_{m}\right)$ [3], sendo, portanto, um parâmetro essencial no dimensionamento da rede. A Figura 2 ilustra os resultados obtidos para capacidade média $G_{m}$ em função do número de gateways, adotando a densidade de nós como parâmetro. Observa-se que, independentemente da densidade de nós, o aumento da densidade de gateways não implica no aumento de $G_{m}$ na mesma proporção. De fato, independentemente da densidade de nós na rede, existe um valor de densidade de gateways a partir do qual um aumento nesta densidade não resulta em um aumento significativo da capacidade por nó. Deste modo, existe um número suficiente de gateways que permite aproximar a capacidade máxima do sistema, o qual pode ser adotado para minimizar os custos de implantação, isto é, uma quantidade ótima de gateways. Isso se deve ao fato de que, ao aumentar a quantidade de gateways, tem-se inicialmente uma redução no tráfego transportado por cada gateway. Porém, a partir de uma certa densidade de gateways, começa a haver sobreposição entre os respectivos domínios de colisão, que impede o aumento da capacidade por nó $G_{m}$.

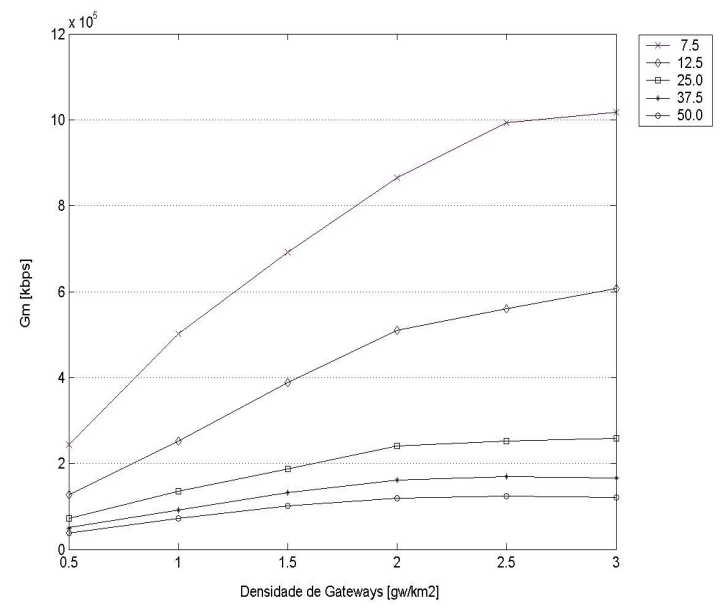

Fig. 2 - Capacidade $G_{m}$ por usuário em função densidade de gateways, para diferentes densidades de nós.

A Figura 3 ilustra a relação entre a capacidade máxima por usuário obtida para o posicionamento e a quantidade ótimos de gateways, sendo esta extraída do gráfico mostrado na Figura 2, e a densidade de nós. A Figura 3 também mostra uma curva de aproximação para a curva obtida pela simulação, dada pela expressão

$$
G_{\max }^{*}=\frac{7,4}{\rho_{n}},
$$

em que $G_{\max }^{*}$ é a capacidade máxima expressa em Mbps e $\rho_{n}$ corresponde à densidade de nós, considerando distribuição uniforme em uma área. Portanto, é possível concluir que $G_{\max }^{*}$ obedece a 
seguinte relação $G_{\max }^{*} \propto 1 / \rho_{n}$, que coincide com a relação descrita em [3] e confirma a consistência dos resultados obtidos.

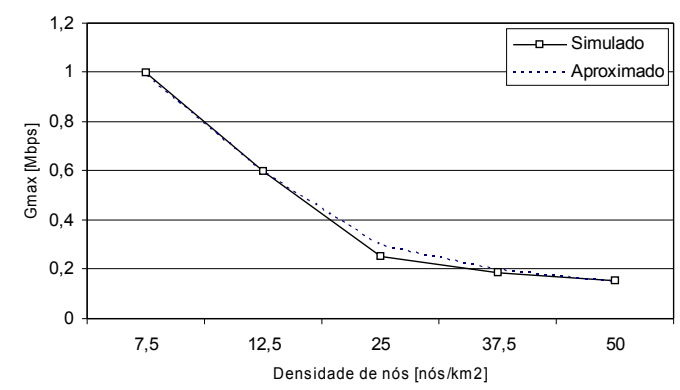

Fig. 3 - Capacidade máxima simulada e aproximada em função da densidade de nós, para número ótimo de gateways.

\section{B. Posicionamento de gateways}

Com base no algoritmo descrito na Seção II, é possível determinar a posição dos gateways que maximiza a banda $G_{m}$ disponível aos usuários. Para exemplificar os resultados gerados, foram considerados cenários com cinco gateways em uma topologia de 50 nós. A Figura 4 ilustra a posição ótima dos gateways (nós 3, 7, 21, 34 e 44). Observa-se que os gateways são posicionados de modo a maximizar o número de nós cobertos e se concentram nas regiões com maior densidade de nós, de modo a minimizar a tendência de maior congestionamento dos domínios de colisão nestas áreas. Conclui-se, portanto, que o posicionamento ótimo dos gateways tende a minimizar a sobrecarga dos domínios de colisão da rede, de modo que as regiões com maior densidade de nós concentrem a maior parte dos gateways disponíveis.

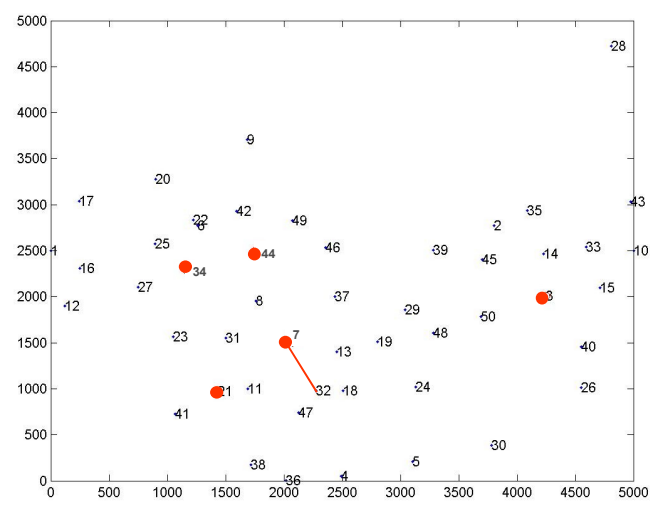

Fig. 4 - Posicionamento ótimo de gateways e enlace gargalo em uma topologia de 50 nós.

C. Identificação de enlaces congestionados

A determinação dos enlaces gargalo constitui uma importante etapa no planejamento e otimização da rede, pois permite determinar com antecedência os prováveis pontos de congestionamento da rede, possibilitando a adoção de medidas para prevenir ou minimizar a ocorrência de congestionamento. Através do modelo de capacidade proposto, são determinados os enlaces com maior volume de tráfego da rede, que representam gargalos para o escoamento do tráfego. Por exemplo, a Figura 4 mostra o enlace de maior congestionamento naquela topologia de 50 nós (linha entre nós 7 e 32). O nó gargalo sempre pertence ao domínio de colisão de um dos gateways, como é característico de redes mesh, visto que o gateway é origem ou destino de todo o tráfego da rede.

\section{Impacto do raio de cobertura}

$\mathrm{O}$ raio de cobertura de cada nó representa um fator de grande relevância no comportamento de uma rede ad hoc ou mesh sem fio, pois tem impacto direto no comprimento das rotas a serem estabelecidas. Visando quantificar este impacto em topologias de menor densidade de nós, adotouse um cenário de simulação com grade de $2,5 \mathrm{~km}$ x 2,5 km e três gateways. Os demais parâmetros são aqueles descritos na Tabela 1. A Figura 5 ilustra os resultados obtidos para topologias de 25 e 50 nós, considerando raios de $300 \mathrm{~m}, 500 \mathrm{~m}$, $750 \mathrm{~m}, 1000 \mathrm{~m}$ e $1250 \mathrm{~m}$. Observa-se que a capacidade por usuário $G_{m}$ diminui com o aumento do raio de cobertura, seguida de uma estabilização. $\mathrm{O}$ aumento do raio de cobertura leva ao aumento do carregamento dos domínios de colisão, devido ao aumento da disputa pelo meio em enlaces CSMA/CA, resultando na redução de $G_{m}$. Por outro lado, o aumento dos raios de cobertura dos nós provoca a redução do comprimento das rotas, o que contribui para o aumento da capacidade $G_{m}$. Para valores elevados de raio de cobertura, observa-se que ocorre uma compensação dos dois efeitos, resultando na estabilidade observada de $G_{m}$. A redução da capacidade em uma topologia com maior densidade de nós tende ser bem mais abrupta, devido à maior intensidade do efeito de congestionamento dos domínios de colisão. De fato, a Figura 5 indica que para raios de cobertura acima de $500 \mathrm{~m}$, a capacidade $G_{m}$ corresponde praticamente à metade da observada para raios abaixo de $500 \mathrm{~m}$ no caso das topologias com 50 nós. No caso das topologias de 25 nós, observa-se uma menor redução da capacidade com o aumento da cobertura. 


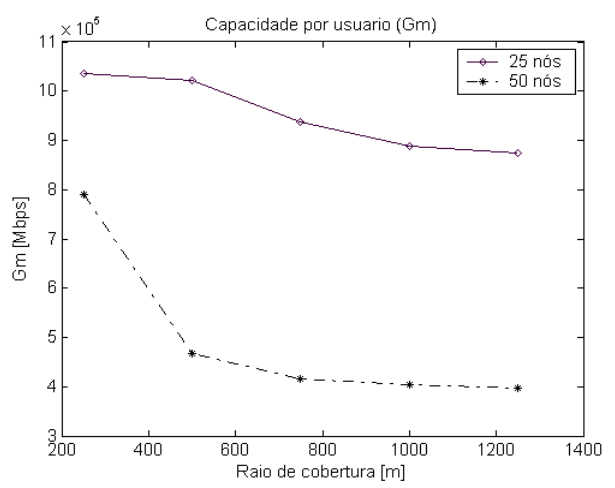

Fig. 5 - Capacidade $G_{m}$ em função do raio de cobertura por nó.

\section{Metodologia de Planejamento}

A partir dos resultados descritos nas seções anteriores, propõe-se nesta seção uma metodologia para o planejamento de redes ad hoc sem fio com foco na maximização da capacidade disponível aos usuários. A metodologia consiste no conjunto de procedimentos descritos a seguir.

Inicialmente, deve-se realizar um levantamento da quantidade e posicionamento dos nós fixos da rede, bem como da área total a ser coberta pela rede. A partir deste levantamento, são obtidas informações como a densidade de nós e a cobertura média necessária para cobrir as regiões de interesse.

Em seguida, os parâmetros que caracterizam o sistema de transmissão a ser adotado são levantados, correspondendo às entradas necessárias para o modelo de simulação de capacidade. São necessários: modelo de propagação, sensibilidade do receptor, raio de cobertura, freqüência de operação, potência de transmissão, ganho da antena de transmissão, ganho da antena de recepção, altura das antenas transmissora, e receptora.

A seguir, estima-se a demanda de tráfego de cada nó da rede, bem como da caracterização dos níveis de qualidade de serviço (QoS), identificando os tipos de tráfego, e definindo critérios de QoS, como perda de pacotes e jitter. Os parâmetros que caracterizam a demanda de tráfego são tipicamente a taxa de chegada de pedidos, taxa de envio e o grau de utilização.

Dimensiona-se então a quantidade e determina-se as posições dos gateways, procurando garantir capacidade e cobertura. A quantidade de gateways para a máxima capacidade é encontrada através da Figura 2. Se esta quantidade não for suficiente para garantir que todos os nós tenham pelo menos uma rota até o gateway, novos gateways são adicionados.

Uma vez definida a quantidade e o posicionamento dos gateways, deve-se estimar a vazão a ser suportada pelos enlaces concentradores (backhaul) de cada gateway. Contudo, observando a relação entre as densidades de nós e gateways, pode-se também obter uma estimativa de vazão média por gateway $\left(S_{G W}\right)$ através da equação:

$$
S_{G W}=\frac{\rho_{n} \cdot v_{u}}{\rho_{G w}} G_{m},
$$

em que $\rho_{n}$ é a densidade de nós, $v_{u}$ é o fator de utilização e $\rho_{G W}$ é a densidade de gateways da topologia.

\section{Conclusões}

As redes ad hoc e mesh sem fio apresentam diversas características que dificultam o dimensionamento de capacidade. Através do modelo de capacidade proposto neste artigo, foram construídas curvas de referência para o dimensionamento da capacidade média por nó em redes ad hoc e mesh sem fio, em função da densidade de nós, da densidade de gateways, do raio de cobertura por nó, do perfil de usuários e dos serviços e do comprimento médio das rotas. Com base no algoritmo proposto, podem ainda ser determinados o posicionamento ótimo dos gateways e os enlaces gargalo de uma topologia específica, bem como os impactos sobre a capacidade média por usuário suportada.

Em comparação com outras abordagens, a metodologia proposta simplifica significativamente a obtenção de estimativas preliminares de capacidade em redes ad hoc e mesh sem fio, na medida em que minimiza a necessidade de simulações pelo emprego de uma família de curvas de capacidade construída, que poderá ser estendida através de novas simulações para ampliar sua abrangência.

\section{REFERÊNCIAS}

[1] P. Gupta e P. R. Kumar, "The Capacity of Wireless Networks", IEEE Trans. on Information Theory, vol. 46, no. 2, pp. 388-404, Março 2000.

[2] J. Li, C. Blake, D. de Couto, H. Lee e R. Morris, "Capacity of Ad hoc Wireless Networks", Proceedings of 7th MobiCom, pp. 61-69, 2001.

[3] J. Jun e M. L. Sichitiu. "The Nominal Capacity of Wireless Mesh Networks". IEEE Wireless Communications, vol. 10, no. 5, pp. 8-14, Outubro 2003.

[4] M. M. Carvalho e J.J. Garcia-Luna-Aceves, "A Scalable Model for Channel Access Protocols in Multihop Ad hoc Networks", Proceedings of the 10th MOBICOM, pp. 330-344, Setembro 2004.

[5] IEEE Standard 802.11-2007. IEEE Standard - Part 11: Wireless LAN Medium Access Control (MAC) and Physical Layer (PHY) Specifications; Junho 2007.

[6] Simulador de Redes NS-2, http://www.isi.edu/nsnam/ns/.

[7] F.L. Figueiredo, Capacidade e Roteamento em Redes Metropolitanas Sem Fio Hibridas, Faculdade de Engenharia Elétrica e de Computação, UNICAMP, Tese de Doutorado; Dezembro 2008. 